\title{
Indicadores
}

\section{SCImago journal \& country rank: un nuevo portal, dos nuevos rankings}

\author{
Por Grupo Scimago
}

Grupo Scimago. "SCImago journal \& country rank: un nuevo portal, dos nuevos rankings". En: El profesional de la información, 2007, noviembre-diciembre, v. 16, n. 6, pp. 645-646.

DOI: 10.3145/epi.2007.nov.11

EL PORTAL SCIMAGO JOURNAL \& COUNTRY RANK nace de la alianza entre la empresa Elsevier B.V. ${ }^{2}$ y el grupo de investigación Scimago ${ }^{3}$. Como resultado de esta sinergia se ha desarrollado una plataforma de indicadores científicos a partir de la información contenida en la base de datos Scopus ${ }^{4}$.

La plataforma toma su nombre del indicador Scimago journal rank $(S J R)$, elaborado por el grupo a partir del algoritmo Page rank, que representa la visibilidad de las revistas contenidas en la base de datos desde 1999 hasta 2006.

\section{Alternativa al JCR}

En la página principal del portal se encuentran dos opciones principales: el ranking de revistas y el de países. Como se puede suponer el portal $S J R$ nace con la vocación de representar una alternativa open access a los productos de Thomson Scientific JCR y ESI .

El ranking de revistas se puede apreciar en la figura 1 (Journal indicators). En él aparecen unos menús desplegables tipo combo a través de los cuales se puede filtrar el ranking por una de las grandes áreas temáticas (27), una categoría temática (295), el país y el año. Hay un combo adicional que permite el ordenamiento del ranking bajo diferentes campos: $S J R$, citas por documento, índice h, título, documen- tos, documentos citables, y total de citas. También existe la posibilidad de recortar el ranking estableciendo un valor mínimo de umbral.

La apariencia del segundo de los rankings se puede apreciar en la figura 2 ("Country Indicators"). Los filtros son similares a los del caso anterior, aunque los campos mostrados son diferentes. Acá se incluyen, además del nombre de cada país, su producción total en número de documentos, la cantidad de citas recibidas, las citas por documentos, y el índice $h$.
Por otro lado, hay que destacar la opción "Journal Search", que permite buscar por una revista en particular, tanto por su título como por su ISSN. El resultado consiste en un resumen de todos los indicadores de esa revista en todo el período de cobertura, así como las representaciones gráficas de algunos de los mismos. Este es un breve informe al que también se puede acceder haciendo click en cada uno de los títulos del ranking de revistas.

En este informe se encuentran los indicadores ya presentados en

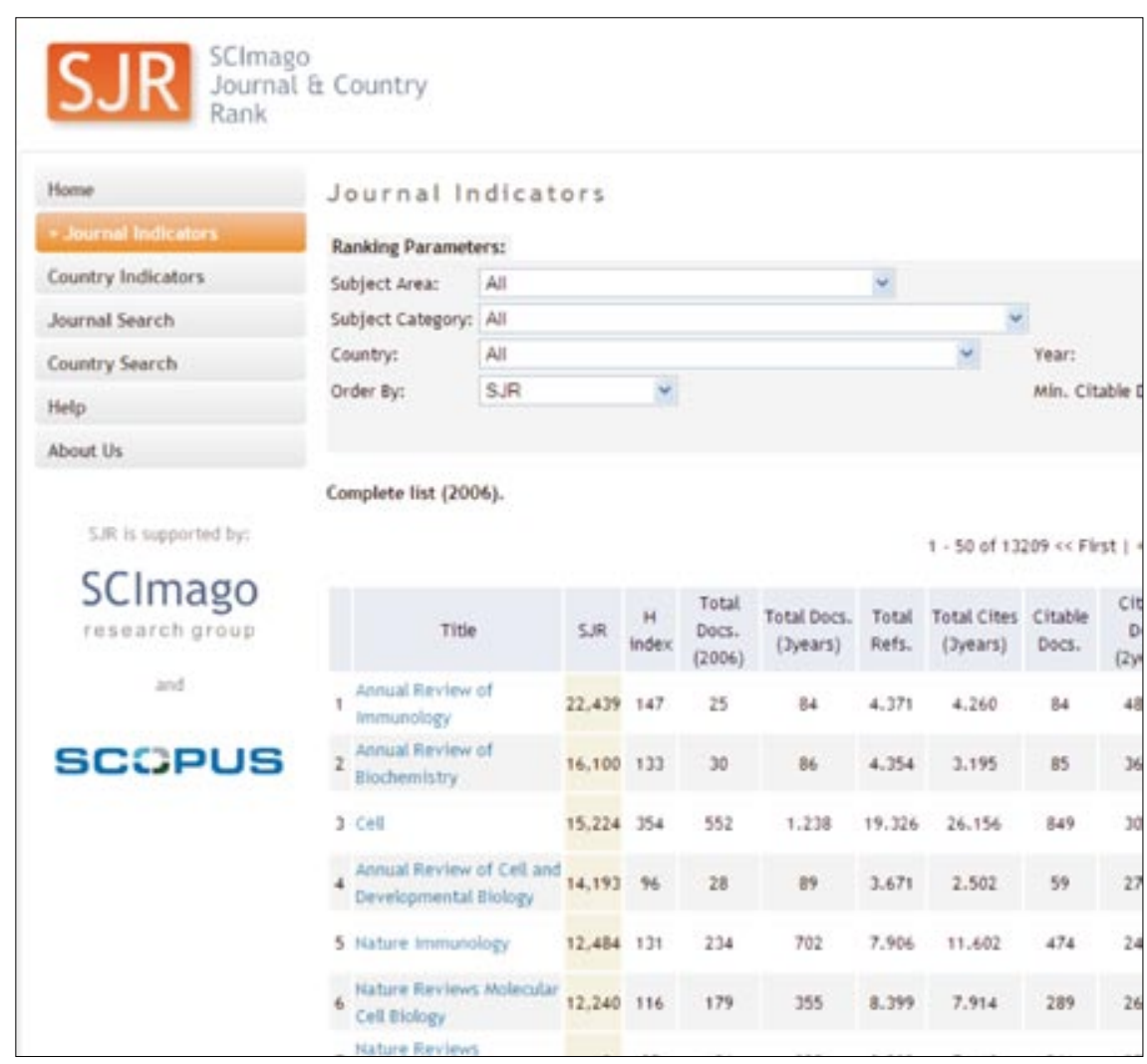

Figura 1. Ranking de revistas 


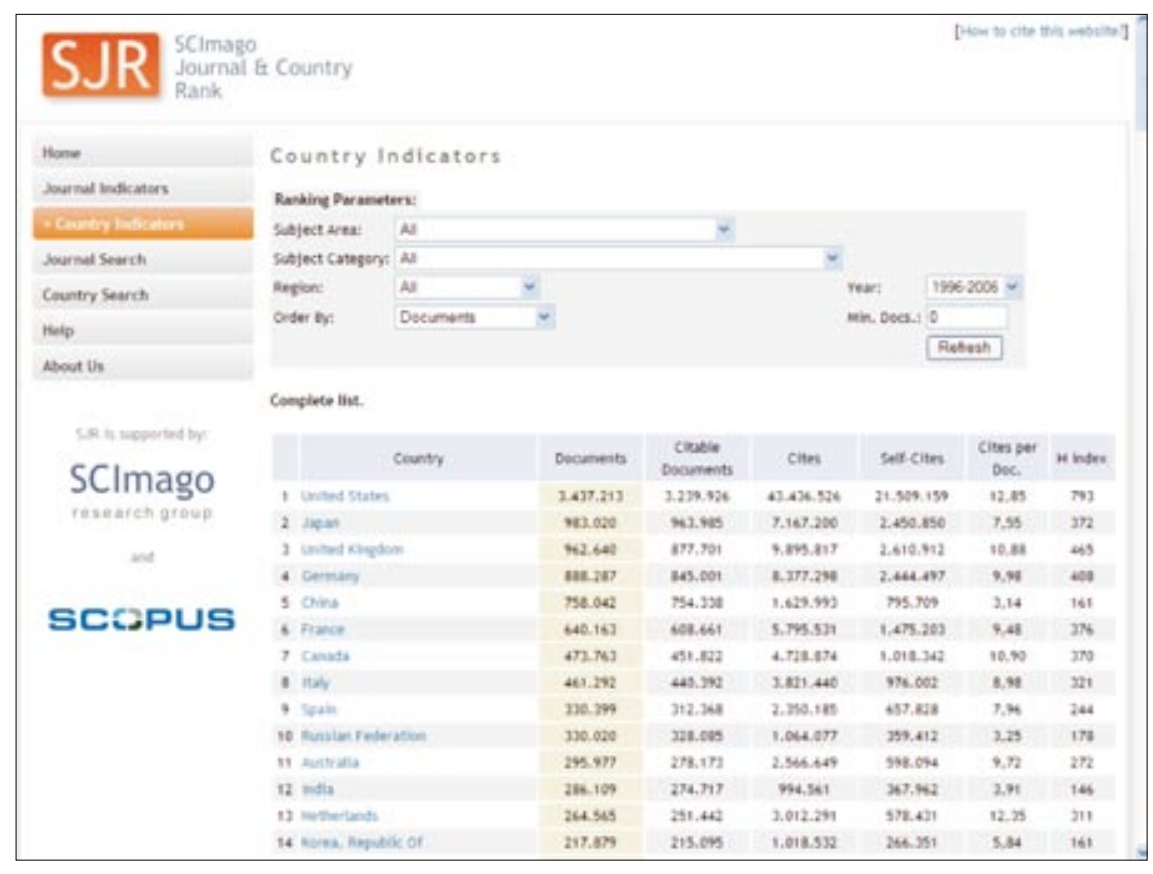

Figura 2. Ranking de países

el ranking principal junto a otros diferentes, discriminados por años. Entre ellos encontramos:

- SJR: es un indicador que expresa el número de enlaces que una revista recibe a través de la citación ponderada de sus documentos en relación con el número de documentos publicados en el año por cada publicación. La ponderación de las citas se hace en función de las que recibe la publicación citante.

- Journal documents: Número de documentos publicados. Para el cálculo de este indicador se tienen en cuenta todos los tipos de documentos incluidos en cada ejemplar de la revista en el año seleccionado.

- Journal documents (3years): Número de documentos publicados en 3 años. Para el cálculo de este indicador se tiene en cuenta el acumulado de los trabajos (cualquier tipo de documento) publicados en los tres años anteriores.

- Journal references: Número total de referencias. Para el cálculo de este indicador se realiza un sumatorio del total de cualquier tipo de referencia bibliográfica incluida en los documentos de la revista en el año seleccionado.
- Total cites (3years): Total de citas realizadas desde la revista en 3 años. Para el cálculo de este indicador se tienen en cuenta los documentos de cualquier tipo aparecidos en el año seleccionado en una publicación y las referencias bibliográficas que han realizado a cualquier documento publicado en los tres años anteriores.

- Autocitas: Número de citas recibidas por la publicación cada año, que proceden de la misma publicación.

- Citable documents: Total de artículos y reviews publicados en tres años. Para el cálculo de este indicador se han tenido en cuenta exclusivamente dos tipologías documentales: los artículos científicos y las revisiones de los tres años anteriores al año seleccionado.

- Cites $x$ document (4years): Promedio de citas por documento en cuatro años. Para el cálculo de este indicador se han tenido en cuenta el número de citas recibidas en los cuatro años anteriores y el número de documentos publicados en el año seleccionado.

- Cites $x$ document (3years): Promedio de citas por documento en tres años. Para el cálculo de este indicador se han tenido en cuenta el número de citas recibidas en los tres años anteriores y el número de documentos publicados en el año seleccionado.

- Cites $x$ document (2years): Promedio de citas por documento en dos años. Para el cálculo de este indicador se han tenido en cuenta el número de citas recibidas en los dos años anteriores y el número de documentos publicados en el año seleccionado

- References $x$ document: Promedio de referencias por documento. Para el cálculo de este indicador se divide el total de referencias incluidas en los documentos de la revista en el año seleccionado entre el total de documentos publicados en ese mismo año.

Por lo que afecta a la función Country search, despliega un conjunto de indicadores científicos absolutos y relativos de cada país representado en la base de datos, con los correspondientes gráficos que facilitan su interpretación.

Como puede apreciarse, el portal presenta una amplia gama de indicadores. Varios de ellos son variaciones en torno al impacto utilizando diferentes ventanas de citación, pero el punto fuerte es el Scimago journal $\operatorname{rank}(S J R)$, que se basa en el algoritmo de Page rank para ponderar las citas que forman parte del cálculo. Sobre este indicador entraremos en detalle en el próximo artículo.

\section{Notas}

$\begin{array}{ll}\text { 1. } & \text { http://www.scimagojr.com/ } \\ \text { 2. } & \text { http://www.elsevier.com/ } \\ \text { 3. } & \text { http://www.scimago.es/ } \\ \text { 4. } & \text { http://www.scopus.com/ } \\ \text { 5. } & \text { http//www.isiknowledge.com/ }\end{array}$

Grupo Scimago (Imago scientiae o visualización de la ciencia) scimago@ugr.es http://www.scimago.es http://www.atlasofscience.net 